Mon. Not. R. Astron. Soc. 000, 000-000 (0000) Printed 30 August $2016 \quad$ (MN LATEX style file v1.4)

\title{
Discovery of two new Fast X-ray Transients with INTEGRAL: IGR J03346+4414 and IGR J20344+3913
}

\author{
V. Sguera ${ }^{1}$, L. Sidoli ${ }^{2}$, A. Paizis ${ }^{2}$, A. J. Bird ${ }^{3}$ \\ 1 INAF, Istituto di Astrofisica Spaziale e Fisica Cosmica, Via Gobetti 101, I-40129 Bologna, Italy \\ 2 INAF, Istituto di Astrofisica Spaziale e Fisica Cosmica, Via E. Bassini 15, I-20133 Milano, Italy \\ 3 School of Physics and Astronomy, University of Southampton, University Road, Southampton, SO17 1BJ, UK
}

Accepted 2016 August 26. In original form 2016 June 1

\begin{abstract}
We report on the discovery of two Fast X-ray Transients (FXTs) from analysis of archival INTEGRAL data. Both are characterized by a remarkable hard X-ray activity above $20 \mathrm{keV}$, in term of duration ( $\sim 15$ and 30 minutes, respectively), peak-flux ( $\left.10^{-9} \mathrm{erg} \mathrm{cm}^{-2} \mathrm{~s}^{-1}\right)$ and dynamic range $(\sim 2400$ and 1360, respectively). Swift/XRT follow-up observations failed to detect any quiescent or low level soft X-ray emission from either of the two FXTs, providing an upper limit of the order of a few times $10^{-12} \mathrm{erg} \mathrm{cm}^{-2} \mathrm{~s}^{-1}$. The main spectral and temporal IBIS/ISGRI characteristics are presented and discussed with the aim of infering possible hints on their nature.
\end{abstract}

Key words: X-rays: transient - X-rays: individual: IGR J03346+4414 - X-rays: individual: IGR J20344+3913

\section{INTRODUCTION}

Since its launch in 2002, the IBIS/ISGRI detector (Lebrun et al. 2003, Ubertini et al. 2003) on board the INTEGRAL observatory (Winkler et al. 2003) has repeatedly proven its suitableness for the investigation of the hard X-ray transient sky at energies above $20 \mathrm{keV}$. Thanks to its powerful combination of large field of view $\left(29^{\circ} \times 29^{\circ}\right.$, partially coded at zero response), fine angular resolution $\left(12^{\prime}\right)$ and good instantaneous sensitivity ( $\sim 7 \mathrm{mCrab}$ at $2 \sigma$ level with an exposure of $\sim 2000 \mathrm{~s}$ ), it has discovered several hundred new hard X-ray transients both on short (hours-days) and long (weeks-months) timescales (e.g. Bird et al. 2016). The great majority of these sources is Galactic in origin since INTEGRAL has spent a considerable fraction of its observational time towards the Galactic plane. In particular, one of the major outcomes has been the discovery of many new fast X-ray transients peculiarly characterized by activity on very short timescale (typically only a few hours as detected above $20 \mathrm{keV}$ ) and subsequently optically identified, decidedly unexpectedly, as supergiant HMXBs (SGXBs). This led to the recognition of a new class of SGXBs which has been named Supergiant Fast X-ray Transients (SFXTs, Negueruela et al. 2006, Sguera et al. 2005, 2006). In fact, before the INTEGRAL era, the 'classical' SGXBs were mistakenly assumed to all be bright persistent X-ray emitters.

Fast X-ray transients (FXTs) are among the most elusive X-ray sources in the sky, they are very difficult to discover because their activity is particularly short and oc- curs at unpredictable locations and times. X-ray instruments having a sufficiently wide field of view and good instantaneous sensitivity, such as IBIS/ISGRI, have the best chance of serendipitously detecting such short duration random events. Due to their elusive nature, a large population of still undetected FXTs could be hidden in our Galaxy; it seems plausible that many such sources wait to be discovered.

Here we report on the analysis of archival INTEGRAL data pertaining to observations of specific regions of the Galactic plane (i.e. Cygnus region and Galactic anticenter) with the aim of finding new FXTs. As result, we report on the discovery of two new such sources which have not been previously detected by any other X-ray telescope. Both are characterized by short (15-30 minutes duration) and bright (average hard X-ray flux of $\sim 10^{-9} \mathrm{erg} \mathrm{cm}^{-2} \mathrm{~s}^{-1}$ ) outbursts as detected by INTEGRAL. Their main spectral and temporal characteristics are presented and discussed with the aim of infering possible hints on their nature.

\section{INTEGRAL}

\subsection{Data analysis}

For our study, we used data collected with the ISGRI detector which is the lower energy layer of the IBIS coded mask telescope. The reduction and analysis of the data have been performed by using the Offline Scientific Analysis (OSA) version 10.1. INTEGRAL observations in each telescope or- 
Table 1. Log of IBIS/ISGRI observations

\begin{tabular}{|c|c|c|c|}
\hline telescope orbit & range time & $\begin{array}{l}\mathrm{ScWs} \\
\left(\mathrm{n}^{\circ}\right)\end{array}$ & $\begin{array}{l}\exp \\
(\mathrm{Ms})\end{array}$ \\
\hline \multicolumn{4}{|l|}{$\begin{array}{l}\text { Anticenter } \\
\left(1 \sim 150^{\circ}\right)\end{array}$} \\
\hline $960-966$ & Aug-Sep 2010 & 569 & $\sim 1$ \\
\hline 1150 & Mar 2012 & 44 & $\sim 0.1$ \\
\hline $1199-1207,1212$ & Aug-Sep 2012 & 655 & $\sim 1.3$ \\
\hline $1254-1255$ & Jan 2013 & 70 & $\sim 0.15$ \\
\hline $1260-1262$ & Feb 2013 & 146 & $\sim 0.3$ \\
\hline \multicolumn{4}{|l|}{$\left(1 \sim 225^{\circ}\right)$} \\
\hline $918-924$ & Apr-May 2010 & 590 & $\sim 1.2$ \\
\hline \multirow[t]{2}{*}{$978-980,982$} & Oct 2010 & 218 & $\sim 0.45$ \\
\hline & & 2,292 & $\sim 4.5$ \\
\hline \multicolumn{4}{|l|}{ Cygnus } \\
\hline $1535-1537,1539$ & Apr-May 2015 & 118 & $\sim 0.25$ \\
\hline $1541-1543$ & May 2015 & 185 & $\sim 0.4$ \\
\hline $1554-1563$ & Jun-Jul 2015 & 464 & $\sim 1.6$ \\
\hline $1600-1603$ & Oct 2015 & 171 & $\sim 0.35$ \\
\hline 1605,1607 & Nov 2015 & 113 & $\sim 0.25$ \\
\hline $1609-1610,1614$ & Nov 2015 & 124 & $\sim 0.25$ \\
\hline $1616,1621,1624$ & Dec 2015 & 123 & $\sim 0.25$ \\
\hline \multirow[t]{2}{*}{$1626-1629$} & Dec 2015-Jan 2016 & 232 & $\sim 0.7$ \\
\hline & & 1,530 & $\sim 4$ \\
\hline
\end{tabular}

bit ("revolution") are divided into short pointings (Science Window, ScW) having a typical duration of $\sim 2000 \mathrm{~s}$.

Our data set consists of targeted observations of the Galactic anticenter region as well as of the Cygnus region. For the Galactic anticenter, two different positions have been observed at $1 \sim 150^{\circ}$ and $1 \sim 225^{\circ}$ respectively, for a total exposure of $\sim 4.5 \mathrm{Ms}$ (see details in Table 1). For the Cygnus region, the data set amounts to a total exposure of $\sim 4 \mathrm{Ms}$ (see Table 1 for details) and consists of recent public ToO observations of V404 Cyg as well as recent public observations targeted on the Cygnus sky region.

We performed an analysis at the ScW level of the full data set to search for new X-ray transient sources detected with a significance greater than at least $6 \sigma$ in a single ScW. The search was initially performed in the energy band 22$60 \mathrm{keV}$; this choice takes into account the evolution of the IBIS/ISGRI energy threshold that occurred from revolution number $\sim 900$ on. When an interesting excess was found, we also checked the detection at higher energies (i.e. 60-100 $\mathrm{keV}$ ) or in other different ranges (i.e. 22-30, 30-60 and 22-40 $\mathrm{keV})$. The sensitivity limit for a persistent source detected at $5 \sigma$ level $(22-60 \mathrm{keV})$ in only one ScW of about $2000 \mathrm{~s} \mathrm{du}-$ ration is $\sim 18 \mathrm{mCrab}$ (Krivonos et al. 2010). We note that this approach is efficient in unveiling FXTs with durations as short as a few tens of minutes, since the search occurs on the same timescale as their outburst activity themselves. Integrating for longer periods just degrades the signal-tonoise ratio of the detection below detectability. The resulting excesses were carefully inspected to identify them as real sources. They were visually examined to ensure an appropriate point spread function and to reject false detections such as ghosts. In order to rule out an artificial nature due to background noise or structures/artifacts, we inspected the overall ScW images rms and checked the residuals maps.
The absence of any known or found systematic effect gives us confidence about the real nature of the two newly discovered FXTs.

A detailed timing and spectral analysis was performed for each newly discovered FXT. Due to possible cross-talk between sources in the same Field of View (FoV), we have also investigated the variability pattern of all other brighter sources in the FoV, besides the two sources of interest. The latter have shown a different time variability, enabling us to conclude that their light curves are uncontaminated and reliable.

The X-ray monitor JEM-X makes observations simultaneously with IBIS/ISGRI, although with a much smaller FoV, providing images in the softer energy band 3-35 $\mathrm{keV}$. Unfortunately both the here reported newly discovered FXTs were outside the JEM-X FoV.

Through the paper, the spectral analysis was performed using XSPEC version 12.9.0 and, unless stated otherwise, errors are quoted at the 90 per cent confidence level for one single parameter of interest.

\subsection{Results}

\subsubsection{IGR J03346+4414}

We report the discovery of a new fast X-ray transient IGR J03346+4414 by analyzing IBIS/ISGRI data of the Galactic anticenter region in revolution number 1261 . The source was detected at a significance of $7.2 \sigma(22-60 \mathrm{keV})$ in a single $\mathrm{ScW}$ (n. 10) starting at 9 Feb 2013 13:04 (UTC) and ending on the same day at 13:37 (UTC).

As can be seen from the extracted $22-60 \mathrm{keV}$ light curve (Fig. 1), the duration of the transient activity is only $\sim 15$ min, it being characterized by a fast rise $(\sim 200 \mathrm{~s})$ followed by a slower decay. At the peak, the source reached a flux of $331 \pm 34$ mCrab $\left(\sim 3.6 \times 10^{-9} \mathrm{erg}^{-2} \mathrm{~s}^{-1}\right)$. We point out that the duration of the transient activity $(\sim 15 \mathrm{~min})$ is significantly shorter than that of the entire ScW number 10 which contain the detection of the source $(\sim 30 \mathrm{~min})$. Bearing this in mind, we performed an imaging analysis with the GTI by considering only the time interval of the transient activity. By doing so, the source was detected at $\sim 12 \sigma$ level (see Fig. 2) in the energy band $22-60 \mathrm{keV}$, while no detection was obtained at higher energies $60-100 \mathrm{keV}$. We note that the source was also significantly detected in both the softer $(22$ $30 \mathrm{keV}, 6.6 \sigma)$ and harder $(30-60 \mathrm{keV}, 9.7 \sigma)$ energy bands.

The best position for the source is $\mathrm{RA}=53^{\circ} .65$ $\left(\mathrm{l}=151^{\circ} .25\right)$ and $\operatorname{Dec}=44^{\circ} .24\left(\mathrm{~b}=-9^{\circ} .47\right)$ with a $90 \%$ confidence error circle radius equal to 2.1 arcminutes. No previously known X-ray source is located inside this error circle according to all the available X-ray catalogs in the HEASARC database. Following the source naming convention, we named this newly discovered IBIS/ISGRI source as IGR J03346+4414.

The IBIS/ISGRI spectrum extracted with the GTI $(22-100 \mathrm{keV})$ is well fitted by a simple power law with $\Gamma=1.99 \pm 0.35\left(\chi_{\nu}^{2}=1.05,9\right.$ d.o.f. $)$ and $18-60 \mathrm{keV}(20-40 \mathrm{keV})$ average flux of $1.9 \times 10^{-9} \mathrm{erg} \mathrm{cm}^{-2} \mathrm{~s}^{-1}\left(1.1 \times 10^{-9} \mathrm{erg} \mathrm{cm}^{-2}\right.$ $\mathrm{s}^{-1}$ ). Fig. 3 shows the data-to-model fit with the corresponding residuals. Alternatively, we have also used a thermal model such as Bremsstrahlung which provides a good fit 


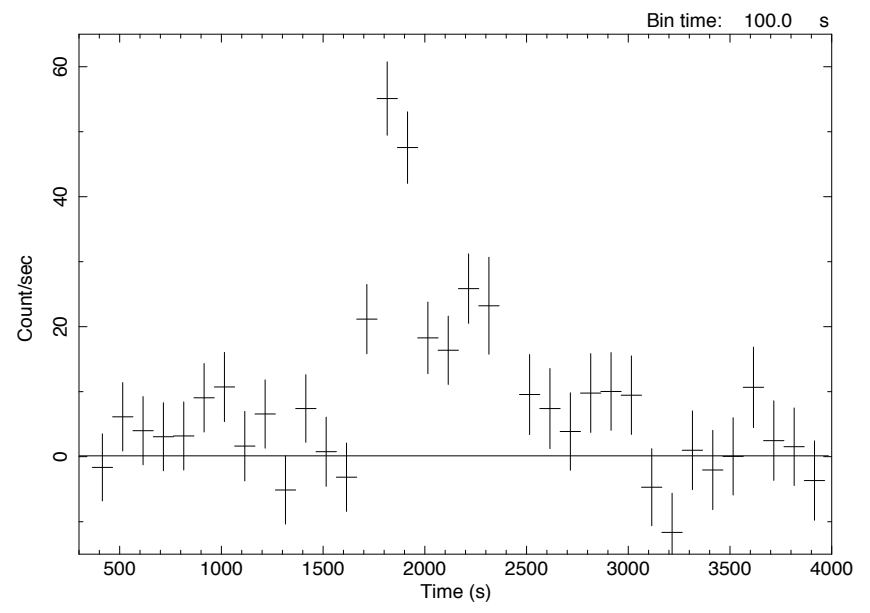

Start Time 16332 13:06:55:185 Stop Time 16332 14:13:35:185

Figure 1. IBIS/ISGRI $22-60 \mathrm{keV}$ light curve (100 s bin time) of the newly discovered source IGR J03346+4414. For display purposes aimed at highlighting the transient nature of the source, the light curve was extracted from the two consecutive ScW number 10 and 11 (revolution 1261) although a source detection was obtained only from ScW number 10.

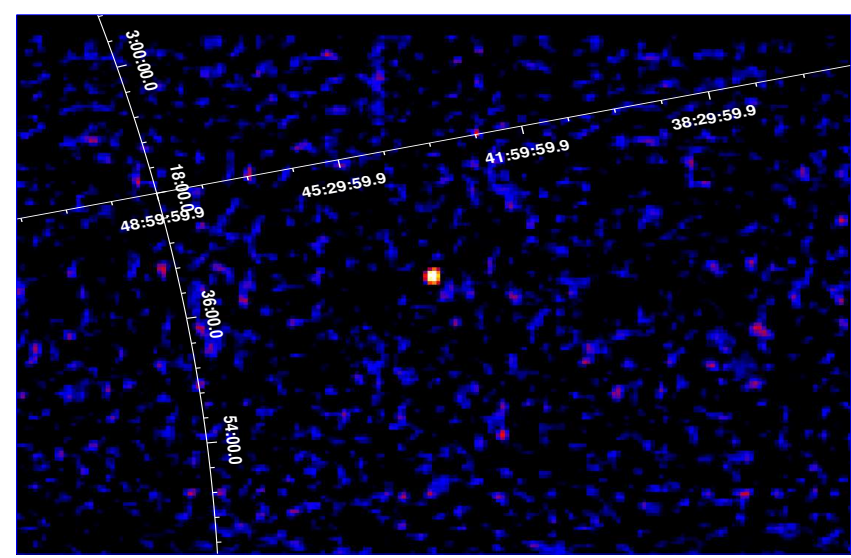

Figure 2. IBIS/ISGRI 22-60 keV significance image (ScW number 10, revolution 1261) of the newly discovered source IGR J03346+4414. It was detected with a significance of $\sim 12 \sigma$.

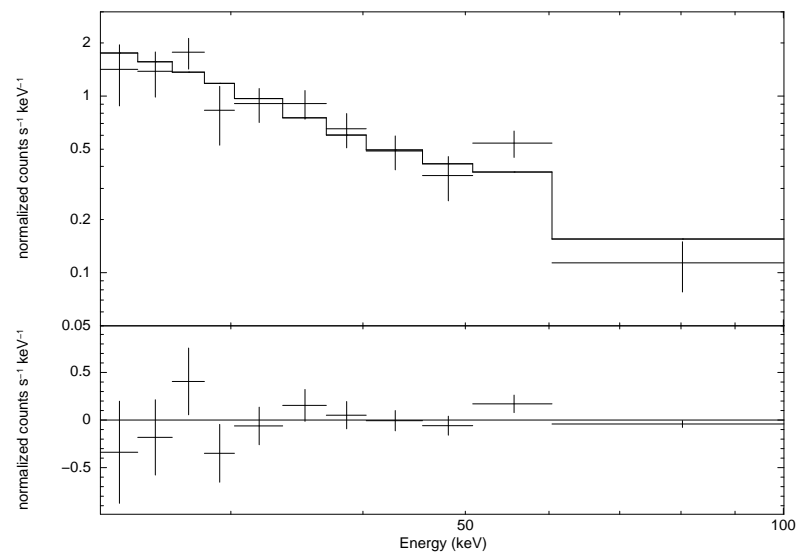

Figure 3. IBIS/ISGRI spectrum of IGR J03346+4414 (extracted with the GTI from ScW number 10) fitted by a power law. The lower panel shows the residuals from the fit.

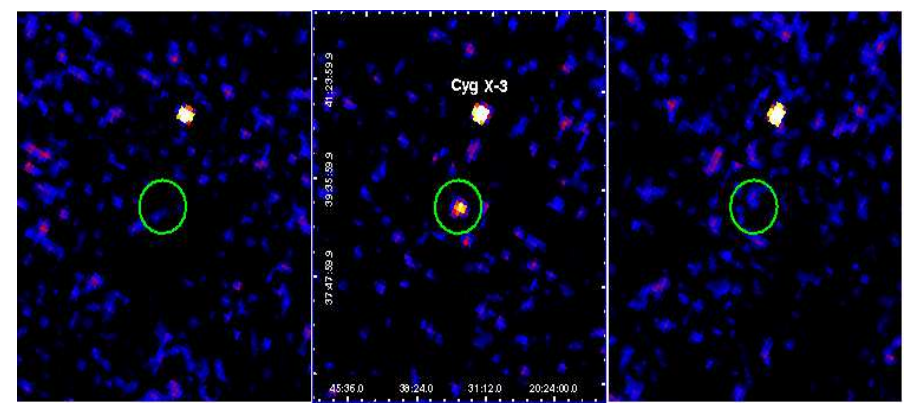

Figure 4. IBIS/ISGRI ScW image sequence $(22-60 \mathrm{keV})$ from number 25 to 27 (revolution 1614) of the newly discovered transient source IGR J20344+3913 (encircled). It was detected in the middle $\mathrm{ScW}$ with a significance of $6.6 \sigma$. The source Cyg X-3 is also detected in the field of view as a bright persistent source.

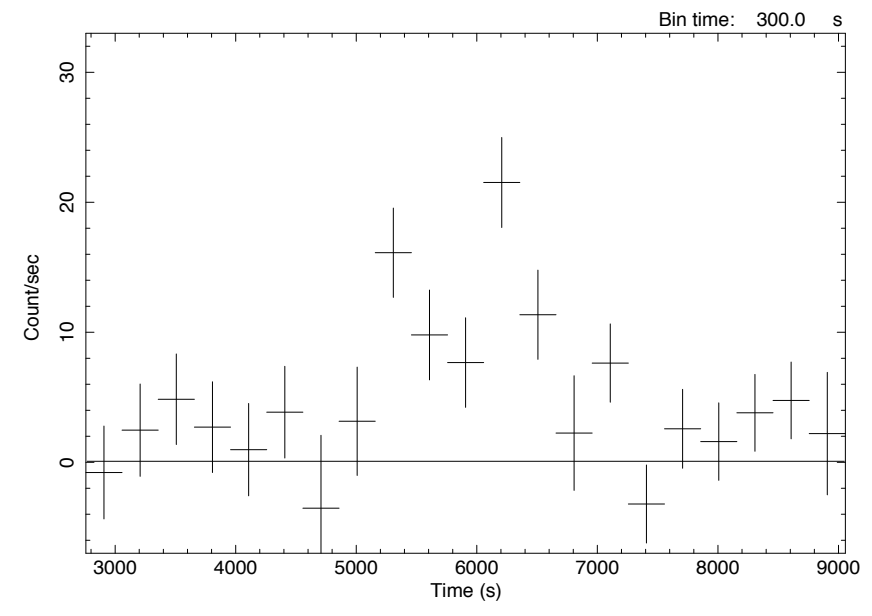

Start Time 17351 1:48:27:186 Stop Time 17351 3:28:27:186

Figure 5. IBIS/ISGRI $22-60 \mathrm{keV}$ light curve (300 s bin time) of the newly discovered source IGR J20344+3913. For display purposes aimed at highlighting the transient nature of the source, the light curve was extracted from the three consecutive ScWs number 25 to 27 (revolution 1614) although a source detection was obtained only in ScW number 26.

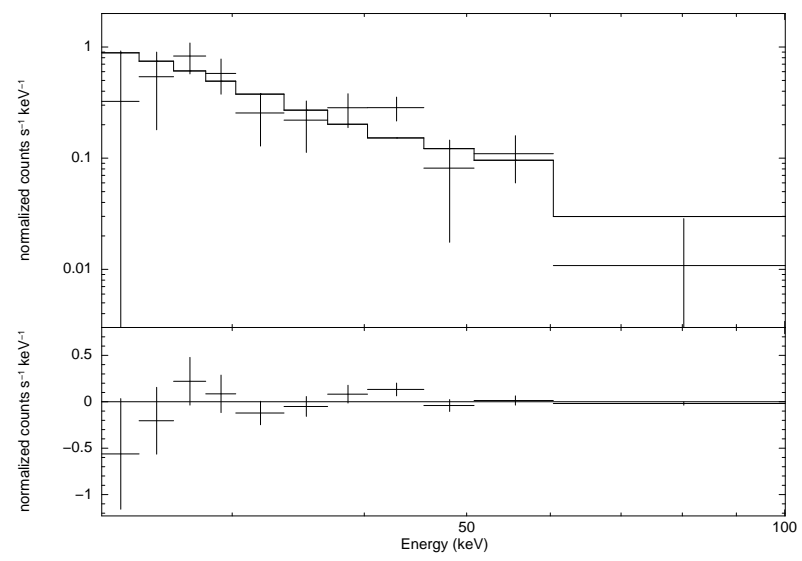

Figure 6. IBIS/ISGRI spectrum of IGR J20344+3913 extracted from Scw number 26 and fitted by a simple power law. The lower panel shows the residuals from the fit. 


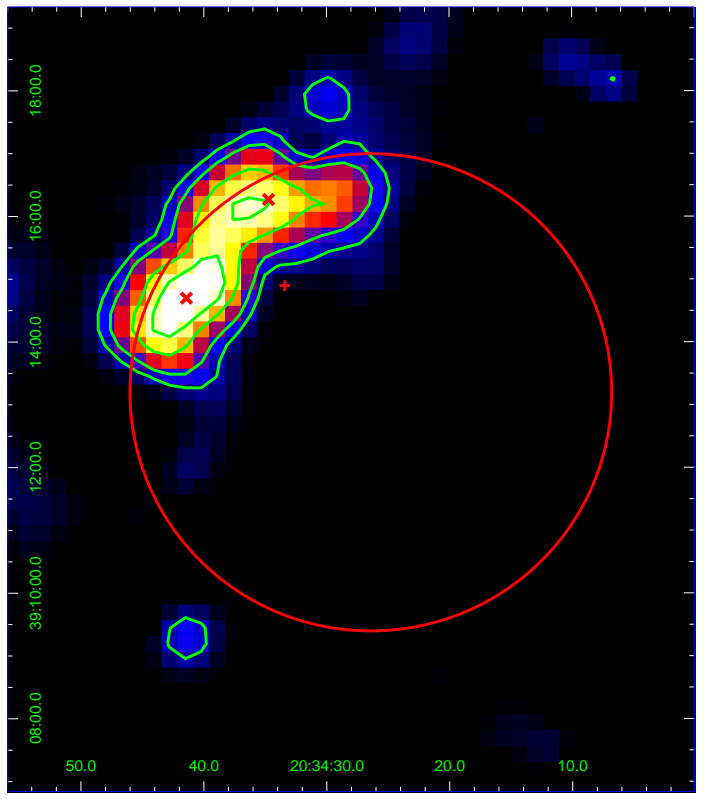

Figure 7. NVSS radio map at $20 \mathrm{~cm}$ (as taken from the NVSS team web page http://www.cv.nrao.edu/nvss/) of the sky region containing the newly discovered INTEGRAL source IGR J20344+3913 (large red error circle). The $\mathrm{x}$ and the cross points mark the position of the NVSS and Green Bank radio sources, respectively.

$\left(\chi_{\nu}^{2}=0.9,9\right.$ d.o.f. $)$, however the temperature value is not well constrained $\left(\mathrm{kT}=70_{-26}^{+66} \mathrm{keV}\right)$.

IGR J03346+4414 is not listed in the latest published IBIS/ISGRI catalog (Bird et al. 2016) despite extensive INTEGRAL coverage of its sky region $(\sim 1 \mathrm{Ms}$ up to revolution number 1000 considered in Bird et al. 2016) and this information can be used to infer an upper limit on its persistent emission. By additionally considering the source exposure from our present dataset $(\sim 2 \mathrm{Ms})$, we can infer a $2 \sigma$ upper limit of $\sim 0.2 \mathrm{mCrab}$ or $1.5 \times 10^{-12} \mathrm{erg} \mathrm{cm}^{-2} \mathrm{~s}^{-1}(20-40 \mathrm{keV})$ for persistent emission. When assuming the source peak flux in the same energy band as measured by IBIS/ISGRI from the outburst reported here, we can infer a dynamic range greater than at least $\sim 2400$.

\subsubsection{IGR J20344+3913}

We report the discovery of a new fast X-ray transient found by analyzing public IBIS/ISGRI data of the Cygnus region in revolution number 1614. It was detected at about $6.6 \sigma$ level $(22-60 \mathrm{keV})$ in a single $\mathrm{ScW}$ (n. 26) starting at 25 Nov 2015 02:19 (UTC) and ending on the same day at 02:52 (UTC). No detection is obtained at higher energies $60-100 \mathrm{keV}$. We note that the source was barely detected in the softer energy band $22-30 \mathrm{keV}(4.3 \sigma)$ while the significance was higher in the harder band $30-60 \mathrm{keV}(5.5 \sigma)$. Fig. 4 shows the IBIS/ISGRI ScW significance image sequence (22-60 keV) from number 25 to 27.

Fig. 5 displays the $22-60 \mathrm{keV}$ light curve which illustrates the fast flaring nature of the source. The duration of its total activity was about 0.5 hour, the source flared up reaching a peak flux of $138 \pm 19 \mathrm{mCrab}\left(\sim 1.5 \times 10^{-9}\right.$ erg $\left.\mathrm{cm}^{-2} \mathrm{~s}^{-1}\right)$.

The extracted IBIS/ISGRI spectrum (22-100 keV) is well fitted by a simple power law with $\Gamma=2.85_{-0.65}^{+0.77}$ $\left(\chi_{\nu}^{2}=1.03,9\right.$ d.o.f. $)$ and $18-60 \mathrm{keV}(20-40 \mathrm{keV})$ average flux of $8.1 \times 10^{-10} \mathrm{erg}^{-2} \mathrm{~s}^{-1}\left(5.1 \times 10^{-10} \mathrm{erg}^{-2} \mathrm{~s}^{-1}\right)$. Fig. 6 shows the data-to-model fit with the corresponding residuals. A thermal Bremsstrahlung model provides a good fit as well, with $\chi_{\nu}^{2}=0.9$ (9 d.o.f.) and $\mathrm{kT}=28.6_{-11.2}^{+24.7} \mathrm{keV}$.

The best source position is $\mathrm{RA}=308^{\circ} .61\left(\mathrm{l}=78^{\circ} .67\right)$ and $\mathrm{Dec}=39^{\circ} .22\left(\mathrm{~b}=-0 .^{\circ} .64\right)$ with a $90 \%$ confidence circle radius equal to 3.8 arcminutes. No previously known X-ray source is located inside this error circle according to all the available X-ray catalogs in the HEASARC database. Following the source naming convention, we named this newly discovered IBIS/ISGRI source as IGR J20344+3913.

In addition we note that, according to all the available radio catalogs in the HEASARC database, the bright radio source GB6 J2034+3914 is located inside the IBIS error circle at a distance of $2^{\prime} .1$. It is listed in the Green Bank radio catalog (Gregory et al. 1996) with a $6 \mathrm{~cm}$ flux of $417 \pm 39$ mJy. Further away, two other bright radio sources (NVSS J203441+391441 and NVSS J203434+391617) listed in the NVSS radio catalog at $20 \mathrm{~cm}$ (Condon et al. 1998) are located inside the IBIS error circle at distance (flux) of $3^{\prime} .3(84 \mathrm{mJy})$ and $3^{\prime} .4(70 \mathrm{mJy})$, respectively. Fig. 7 shows the IBIS/ISGRI error circle superimposed on the NVSS $20 \mathrm{~cm}$ radio map of the sky region. The $\mathrm{x}$ and the cross points mark the position of the NVSS and Green Bank radio sources, respectively. We note that the two NVSS radio sources are close $\left(\sim 2^{\prime}\right.$ from each other) and represent the brightest peak of an extended radio emission as detected at $20 \mathrm{~cm}$. The position of the Green Bank radio source (cross point) is equally distant $\left(\sim 1^{\prime} .4\right)$ from the two NVSS radio sources. We point out that the $6 \mathrm{~cm}$ Green Bank radio survey had an angular resolution of about $3^{\prime} .5$ and it detected sources with a positional uncertainty as high as $1^{\prime}$. Bearing this in mind, it cannot be excluded that during its survey the Green Bank telescope detected a radio source (GB6 J2034+3914) which is actually the same source as one or both the NVSS radio objects (NVSS J203441+391441 and NVSS J203434+391617). This possibility is further corroborated by the fact that GB6 J2034+3914 is listed in the Green Bank catalog as flagged, to indicate that there is either significant extension to the source or that the source is partially resolved blend of two or more sources.

IGR J20344+3913 is not listed in the latest published IBIS/ISGRI catalog (Bird et al. 2016) despite extensive INTEGRAL coverage of its sky region $(\sim 3 \mathrm{Ms}$ up to revolution 1000 considered in Bird et al. 2016), this information can be used to infer an upper limit on its persistent emission. By additionally considering the source exposure from our present dataset ( $\sim 4 \mathrm{Ms}$ ), we can infer a $2 \sigma$ upper limit of $\sim 0.15 \mathrm{mCrab}$ or $1.1 \times 10^{-12} \mathrm{erg} \mathrm{cm}^{-2} \mathrm{~s}^{-1}(20-40 \mathrm{keV})$ for persistent emission. When assuming the source peak flux in the same energy band as measured by IBIS/ISGRI from the outburst reported here, we can infer a dynamic range greater than at least $\sim 1360$. 
Table 2. Swift/XRT (PC) observation log of the two ToOs targeted on the IBIS/ISGRI sky positions.

\begin{tabular}{ccccc}
\hline \hline Target & Obs. ID & Start Time & End Time & XRT/PC Exposure Time (s) \\
\hline IGR J03346+4414 & 00034387001 & 2016-03-06 00:36:28 & 2016-03-07 00:52:53 & 2932 \\
IGR J20344+3913 & 00034386001 & 2016-03-04 01:51:47 & 2016-03-04 03:43:54 & 2208 \\
\hline
\end{tabular}

Table 3. Swift/XRT results of the two ToOs centered on the two IBIS/ISGRI sources, the last row reports the properties of the Xray source detected in the IGR J20344+3913 field: $3 \sigma$ upper limits to the Swift/XRT count rate $(0.3-10 \mathrm{keV})$ of the two IBIS/ISGRI sources are listed in column 2, calculated for a non-detection within the 90\% IBIS error circle. Assuming the average Galactic column density towards the targets (column 3, Dickey \& Lockman 1990), we list in column 4 and 6 the $3 \sigma$ upper limits to the fluxes corrected

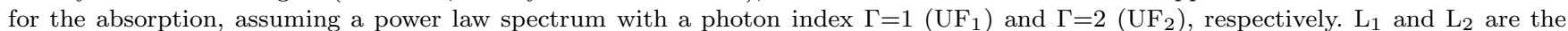
correspondent luminosities, at $10 \mathrm{kpc}$ (IGR J03346+4414) and $5 \mathrm{kpc}$ (IGR J20344+3913 and the new XRT source).

\begin{tabular}{|c|c|c|c|c|c|c|}
\hline $\mathrm{Src}$ & $\begin{array}{l}\text { XRT Rate }(0.3-10 \mathrm{keV}) \\
\left(\text { counts s}{ }^{-1}\right)\end{array}$ & $\begin{array}{c}\mathrm{N}_{\mathrm{H}} \\
\left(\mathrm{cm}^{-2}\right)\end{array}$ & $\begin{array}{c}\mathrm{UF}_{1}(0.3-10 \mathrm{keV}) \\
\left(\mathrm{erg} \mathrm{cm}-2 \mathrm{~s}^{-1}\right)\end{array}$ & $\begin{array}{c}\mathrm{L}_{1} \\
\left(\operatorname{erg~s}^{-1}\right)\end{array}$ & $\begin{array}{l}\mathrm{UF}_{2}(0.3-10 \mathrm{keV}) \\
\left(\operatorname{erg~cm} \mathrm{cm}^{-2} \mathrm{~s}^{-1}\right)\end{array}$ & $\begin{array}{c}\mathrm{L}_{2} \\
\left(\operatorname{erg~s}^{-1}\right)\end{array}$ \\
\hline IGR J03346+4414 & $<1.3 \times 10^{-2}$ & $2.7 \times 10^{21}$ & $<1.1 \times 10^{-12}$ & $<1.3 \times 10^{34}$ & $<8.4 \times 10^{-13}$ & $<1.0 \times 10^{34}$ \\
\hline IGR J20344+3913 & $<1.7 \times 10^{-2}$ & $1.2 \times 10^{22}$ & $<2.1 \times 10^{-12}$ & $<6.2 \times 10^{33}$ & $<2.2 \times 10^{-12}$ & $<6.5 \times 10^{33}$ \\
\hline new XRT source & $(8.8 \pm 2.2) \times 10^{-3}$ & $1.2 \times 10^{22}$ & $1.07 \times 10^{-12}$ & $3.2 \times 10^{33}$ & $1.12 \times 10^{-12}$ & $3.3 \times 10^{33}$ \\
\hline
\end{tabular}

\section{SOFT X-RAY OBSERVATIONS}

We requested ToO observations at the sky positions of the two new IBIS/ISGRI sources with the Swift satellite, to look for possible soft X-ray counterparts, and eventually refine their celestial coordinates. Indeed, to date no soft X-ray observations (except the ROSAT All Sky Survey) have ever covered the IBIS/ISGRI sky positions. The two ToOs were performed between 2016 March 4 and 7 (see Table 2 for the summary $\log$ ) in photon counting (PC) mode. We used HEASOFT version 6.18 and the most up to date calibration files to perform the Swift data reduction and analysis.

\section{$3.1 \quad$ Swift results}

We extracted Swift/XRT sky images in the energy range 0.3$10 \mathrm{keV}$. Using ximage we searched for soft excesses by means of the tool detect. No excesses with a minimum signal-tonoise ratio $(s n r)$ of 3 were found inside the two IBIS/ISGRI error circles. Therefore, we estimated $3 \sigma$ upper limits to the soft flux, for a source centroid variable within their error circles, using the tool sosta (see Table 3 for these results), which makes use of a local estimate of the background. Using WebPIMMS and adopting the average absorbing column density along the line of sight (Dickey \& Lockman 1990) we estimated the $3 \sigma$ upper limits (UF) to the soft fluxes corrected for the absorption, adopting a power-law model with two values for the photon index $(\Gamma=1$ and $\Gamma=2)$. The results are reported in Table 3 , where also the $3 \sigma$ upper limits to the X-ray luminosity are listed. For IGR J20344+3913 which is located in the Cygnus region, we assumed a distance of $5 \mathrm{kpc}$ in the hypothesis that it resides in the Cygnus spiral arm (Hachisuka et al. 2009, Kothes et al. 2014). For IGR J03346+4414, we assumed a canonical value of $10 \mathrm{kpc}$ for the distance since no assumption can be made on its location arm.

No other excesses were found in the whole Swift/XRT field-of-view of the two observations, except for the one targeted on IGR J20344+3913, where a faint source $(s n r=3.6)$ was detected at the following sky position: R.A. (J2000) = 20:33:57.3, Dec $(\mathrm{J} 2000)=+39: 15: 11.6$ (error radius of $7^{\prime \prime}$, estimated with the tool xrtcentroid). The $0.3-10 \mathrm{keV}$ net source count rate is $(8.8 \pm 2.2) \times 10^{-3}$ counts s$^{-1}$ (corrected for the PSF, sampling dead time and vignetting). In Table 3 (last row) we report on the estimated fluxes and luminosities for a power-law continuum. This faint X-ray source lies about 6 ' from IGR J20344+3913 (well outside its error circle radius of $3.8^{\prime}$ ), so it cannot be associated with it. A search in the on-line catalogues at all wavelengths revealed that this new XRT source is positionally coincident (offset of $6^{\prime \prime}$ ) with the source USNO-B1.0 1292-0414079 (also consistent with 2MASS $20335767+3915073$ ), showing the following magnitudes: $\mathrm{J}=10.925 \pm 0.025, \mathrm{H}=10.445 \pm 0.021$, $\mathrm{K}=10.289 \pm 0.014$ (2MASS catalogue, Cutri et al. 2003) and $\mathrm{B} 1=13.39, \mathrm{R} 1=12.10, \mathrm{~B} 2=13.90, \mathrm{R} 2=11.87, \mathrm{I}=11.21$ (USNO-B1.0 catalogue, Monet et al. 2003). In the Guide Star Catalogue (GSC2.3, Lasker et al. 2008) it is classified as a star with a magnitude $\mathrm{V}=12.47$. Fig. 8 shows a close-up view of both XRT and UVOT images, around this faint X-ray source. Adopting to its visual magnitude and an X-ray flux of $10^{-12} \mathrm{erg} \mathrm{cm}^{-2} \mathrm{~s}^{-1}$, we calculated a ratio $\log \left(\mathrm{f}_{X} / \mathrm{f}_{V}\right)=-1.64$, which is compatible with a stellar origin (Maccacaro et al. 1988), although the X-ray flux is likely overestimated because of the assumption of the power-law continuum (Table 3). If the X-ray source is really associated with this star, it should be located within $\sim 100 \mathrm{pc}$, to enable a low X-ray luminosity of the order of $1.3 \times 10^{30} \mathrm{erg} \mathrm{s}^{-1}$. 


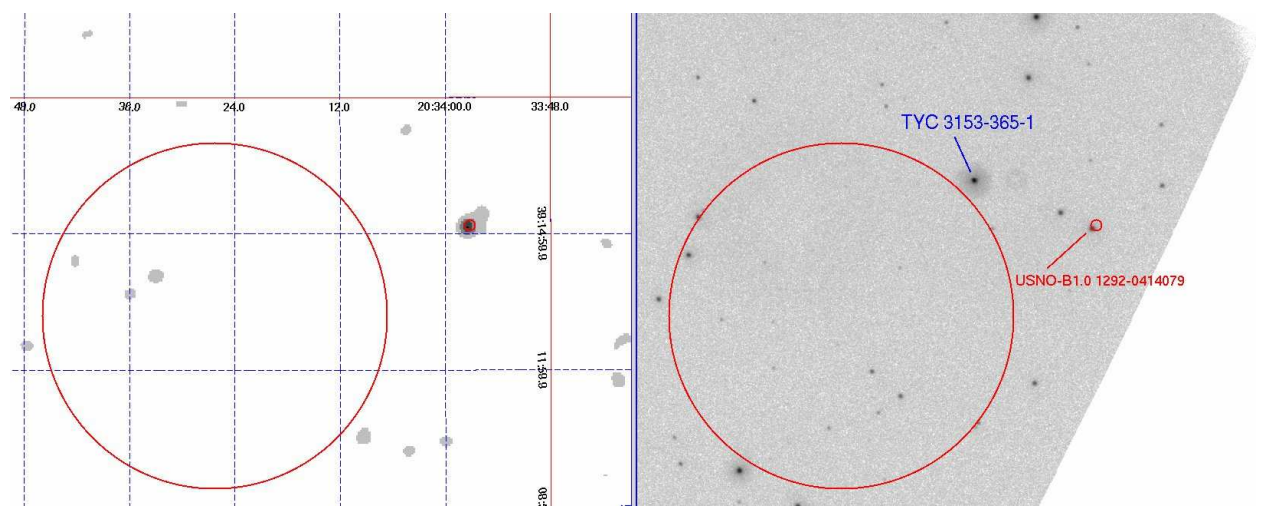

Figure 8. Swift/XRT observation targeted on the source IGR J20344+3913. Left panel: Close-up view of the center of the XRT observation: the large big red circle marks the $3.8^{\prime}$ error radius of IGR J20344+3913, while the small red circle (radius, $\mathrm{R}=7^{\prime \prime}$ ) indicates the only source detected during this XRT snapshot (the XRT image has been smoothed with a Gaussian filter only for graphical reasons, to better show the counts excess). Right panel: UVOT image, where the same XRT error regions have been displayed in red. The XRT source is positionally coincident (within $6^{\prime \prime}$ ) with the star USNO-B1.0 1292-0414079 (2MASS 20335767+3915073, see text). Also the bright star TYC 3153-365-1 (VT mag=11.686 \pm 0.115 ) is indicated, to better identify the stellar field.

\section{DISCUSSION}

From analysis of archival INTEGRAL data, we have presented IBIS/ISGRI results on the two newly discovered FXTs IGR J03346+4414 and IGR J20344+3913. They have been detected only once and never again on February 2013 and November 2015 respectively, being characterized by flaring activity lasting in the range 15-30 minutes and having average flux of the order of $\sim 10^{-9} \mathrm{erg} \mathrm{cm}^{-2} \mathrm{~s}^{-1}$ (22-60 keV). Recent ToO observations performed with the Swift/XRT satellite failed to detect any quiescent or low level soft X-ray emission from either of the two FXTs, providing stringent $3 \sigma$ upper limits of the order of a few times $10^{-12} \mathrm{erg} \mathrm{cm}^{-2} \mathrm{~s}^{-1}(0.3-10 \mathrm{keV})$. Similarly, we obtained stringent upper limits on their persistent emission above 20 $\mathrm{keV}$ as well (of the order of $\sim 10^{-12} \mathrm{erg} \mathrm{cm}^{-2} \mathrm{~s}^{-1}$ ) allowing us to derive high dynamic range values of 1360 and 2400, respectively. After discovering the two FXTs analyzing our IBIS/ISGRI dataset (revolution number in the range 15351629 for the Cygnus region and 918-1262 for the Galactic anticenter), we performed a search at the known source coordinate position for additional possible flares detected in the entire IBIS/ISGRI archive (Paizis et al. 2013) covering revolution number in the range $25-1519$ ( $\sim 12$ years of data), i.e. prior to our current dataset. For both FXTs, no detection was found at $\mathrm{ScW}$ level above a significance value of $7 \sigma$ in several different energy bands (17-50 keV, 17-30 $\mathrm{keV}, 30-50 \mathrm{keV})$. The exposure times obtained from such entire archive revolution 25-1519 were of the order of $\sim 12$ Ms (IGR J20344+3913) and $\sim 5$ M s (IGR J03346+4414).

Although we are aware that all the information reported here is insufficient to firmly identify the nature of the two FXTs, in the following they can be used to obtain some hints and/or indications on the most likely class of X-ray sources to which they could belong to.

\subsection{IGR J03346+4414}

At first glance, its location off the Galactic plane $\left(\mathrm{b} \sim-9.5^{\circ}\right)$ would naturally imply an extragalactic blazar nature, how- ever this scenario is strongly weakened by the lack of a strong radio source inside the error circle according to all the available catalogs in the HEASARC and NED database.

On the other hand, the fast X-ray transient behavior, the IBIS/ISGRI spectral shape, the high dynamic range, all are compatible with a Galactic SFXT scenario. The location of the source off the Galactic plane is apparently irreconcilable with a HMXB nature since such sources are typically located on the Galactic plane in star forming regions. However we note that, although very unlikely, some HMXBs could eventually be located off the plane due to an effect of perspective if they are particularly nearby ( i.e. distance $<$ $1 \mathrm{kpc}$ ) much like the case of the Be HMXB $\chi$ Per located at $\mathrm{b} \sim-17^{\circ}$. Under this assumption, IGR J03346+4414 would be characterized by a $22-60 \mathrm{keV}$ average (peak) outburst luminosity of $<2 \times 10^{35} \mathrm{erg} \mathrm{s}^{-1}\left(<4 \times 10^{35} \mathrm{erg} \mathrm{s}^{-1}\right)$ while the Swift/XRT flux upper limit would translate into an average luminosity of $<1 \times 10^{32} \mathrm{erg} \mathrm{s}^{-1}$. All such values are quite low for a SFXT scenario although they could eventually be still compatible with the weakest hard X-ray flares detected from typical SFXTs and with the rare quiescent state observed for SFXTs, respectively. According to the HEASARC database, 40 2MASS sources and 121 USNOB1.0 optical stars are located within the $2 .^{\prime} 1$ error circle radius of IGR J03346+4414. The brightest near infrared (NIR) source is 2MASS 03344236+4414207 ( $\mathrm{J}=11.716 \mathrm{mag}$, $\mathrm{H}=11.126 \mathrm{mag}, \mathrm{K}=11.005 \mathrm{mag})$. If we assume a B-type supergiant nature for it to reconcile with both the 2MASS magnitudes and observed colors, it should be affected by an extinction, Av, of 5 magnitudes and it should be located at a large distance of $\sim 20 \mathrm{kpc}$ (which is very unlikely). A much nearer main sequence B star (about $5 \mathrm{kpc}$ ) affected by a similar extinction could account for both the IR colors and the faint IR magnitudes, as well. This latter possibility would indicate a Be HMXB nature. Unfortunately the high number of NIR/optical sources within the large ISGRI error circle prevents us to pinpoint the correct counterpart and we cannot constrain the source nature. To this aim, it is mandatory to reduce the value of the error circle radius to arcsecond size. 
An alternative and interesting Galactic scenario is that involving nearby flare stars. In this case flaring activity originates in main sequence or pre-main sequence K-M stars, from plasma magnetically confined in compact structures in the stellar outer atmosphere and heated at very hot temperatures of the order of $10^{7}-10^{8} \mathrm{~K}$ (see Maggio 2008 for a review). Stellar flares in the soft X-ray band $0.2-10 \mathrm{keV}$ have been extensively studied by essentially every major Xray mission. Measured soft $\mathrm{X}$-ray fluxes can be as high as $\sim 10^{-8} \mathrm{erg} \mathrm{cm}^{-2} \mathrm{~s}^{-1}$ with corresponding X-ray luminosities as high as $\sim 10^{33} \mathrm{erg} \mathrm{s}^{-1}$ for very nearby stars (i.e. distances typically in a range from a few parsec to a few hundreds of parsec). On the contrary, in the hard X-ray domain $(\mathrm{E}>20 \mathrm{keV})$ only a few firm events have been detected by the PDS onboard BeppoSAX (Schmitt \& Favata 1999, Pallavicini et al. 2000), Swift/BAT (Osten et al. 2007, 2010, 2016, Copete et al. 2008) and INTEGRAL/IBIS (Bird et al. 2016). This is because the hard X-ray emission is much less intense than soft X-ray (e.g. by a factor of $\sim 10^{5}$ ) and the available hard X-ray instruments have too limited sensitivity, such that only the largest flares (which are intrinsically rare) can be unambiguously detected. Hard X-ray emission from stellar flares is mainly thermal in origin, being best interpreted by single or multi temperature models of an optically thin plasma (e.g. Bremss, APEC, MEKAL in $\mathrm{XSPEC}$ terminology). However non thermal emission is also expected in the form of an additional power law tail component which is weak and very difficult to disentangle from the thermal component, in fact to date non thermal hard X-ray emission from stellar flares has escaped firm detections. If we consider the Swift mission, to date it has detected $\sim 5$ stellar flares which were bright enough to trigger BAT (i.e. flux > $3 \times 10^{-9} \mathrm{erg} \mathrm{cm}^{-2} \mathrm{~s}^{-1}$ in the $15-50 \mathrm{keV}$ band). In most cases only high temperature thermal emission was detected with values as high as $\sim 3 \times 10^{8} \mathrm{~K}$, however in at least one case a possible excess over the thermal emission component was observed and interpreted as most likely non thermal emission (Osten et al. 2007). The typical durations of the events detected by BAT is from several minutes to several hours, conversely in the soft X-ray band stellar flares can last even much longer (from few hours to few days). The duration of the flare detected by INTEGRAL from IGR J03346+4414 ( $\sim 15$ minutes $)$ is compatible with that of stellar flares as typically observed in the hard X-ray band, moreover its location off the Galactic plane could be eventually explained by its nearby location. If we assume a reasonable distance of $100 \mathrm{pc}$, the corresponding $22-60 \mathrm{keV}$ peak (average) $\mathrm{X}$ ray luminosity would be $\sim 4 \times 10^{33} \mathrm{erg} \mathrm{s}^{-1}\left(\sim 2 \times 10^{33} \mathrm{erg} \mathrm{s}^{-1}\right)$ which is consistent with expectations for typical stellar flares detected in the hard X-ray band. As comparison, the only stellar flare detected by INTEGRAL/IBIS to date is that from the flare star GT Mus (172 parsec distance) which reached a $20-40 \mathrm{keV}$ average luminosity of $\sim 1.2 \times 10^{33} \mathrm{erg}$ $\mathrm{s}^{-1}$ (Bird et al. 2016, 2010). As for the spectral shape, the hard X-ray spectrum of IGR J03346+4414 is well fitted by a power law model with a soft photon index of $\sim 2$, consistent with expectations of typical stellar flare detected in the hard X-ray band. Alternatively, a single temperature model (Bremsstrahlung) also provided a good fit with a particularly high temperature of $\mathrm{kT} \sim 70 \mathrm{keV}$ or $\mathrm{T} \sim 8 \times 10^{8} \mathrm{~K}$, although the nominal value is poorly constrained in the range $\sim 44-135 \mathrm{keV}$ or $(5-15) \times 10^{8} \mathrm{~K}$. In addition here we note that an equally good fit $\left(\chi_{\nu}^{2}=0.9,9\right.$ d.o.f. $)$ was also achieved with a APEC thermal model, the temperature was not constrained but its nominal value $(\sim 65 \mathrm{keV})$ was consistent with the Bremsstrahlung measurement. Both temperature values would clearly be representative of an "superhot" thermal plasma (Hudson \& Nitta 1996). This high plasma temperature value could eventually be deemed unlikely and rejected as unphysically high, but we note that temperatures as high as $\sim 10^{8} \mathrm{~K}$ have been measured from many previous large stellar flares. For example, a temperature of $\sim 3 \times 10^{8} \mathrm{~K}$ has been measured with Swift/BAT from the source II PEG during a huge flare (Osten et al. 2007) as well as from the source DG CVn (Osten et al. 2016). In addition, the Chandra Orion ultra deep project (Getman et al. 2008) allowed the detection of several stellar flares with peak plasma temperatures in excess of $\sim 2 \times 10^{8} \mathrm{~K}$, with the most extreme case even reaching a value of $\sim 7 \times 10^{8} \mathrm{~K}$. In our specific case, the high measured temperature of $\sim 8 \times 10^{8} \mathrm{~K}$ could be explained either by the hard tail of a "superhot" thermal plasma (among the hottest thermal emission from possibly a flaring star, to our knowledge), or by a non-thermal component in addition to a thermal plasma emission which we cannot constrain, given the high energy spectrum available $(\mathrm{E}>20 \mathrm{keV})$.

\subsection{IGR J20344+3913}

The fast X-ray transient behavior, IBIS/ISGRI spectral shape (e.g. Sguera et al. 2008), location on the Galactic plane and high dynamic range strongly suggest that IGR J20344+3913 is a Galactic SFXT. The 0.3-10 keV luminosity upper limit $\left(\sim 6 \times 10^{33} \mathrm{erg} \mathrm{s}^{-1}\right.$ at $5 \mathrm{kpc}$ distance) is compatible with the source being a SFXT in the classical intermediate X-ray state or during the rarer quiescence. The inferred duty cycle is remarkably low $(\sim 0.01 \%)$ since strong flaring activity has only been detected by IBIS/ISGRI once despite its sky region having been extensively observed with a total exposure of $\sim 16 \mathrm{Ms}$ to date (i.e. obtained by summing $12 \mathrm{Ms}$ from entire archive revolution $25-1519$ and 4 Ms from our current dataset revolution 1535-1629). Considering that classical SFXTs are characterized by a duty cycle value typically in the range $(0.1-5) \%$ when observed above $20 \mathrm{keV}$ by INTEGRAL (Paizis \& Sidoli 2014), the value of IGR J20344+3913 is significantly lower than this range. On one side this could cast some doubts on its SFXT nature, on the other side it could be due to a marked scarcity of detected outbursts which could have been missed by INTEGRAL eventually because of an observational effect, i.e. the source's particularly large distance would allow the detection of only the brightest (and rarest) outbursts, much like the case of the candidate SFXT IGR J18462-0311 possibly located at a distance of $11 \mathrm{kpc}$ (Sguera et al. 2013). If we follow this line of reasoning and assume a large distance for IGR J20344+3913 in the range $5-10 \mathrm{kpc}$ (this being the value derived from the reasonable assumption that it resides in the Cygnus spiral arm), then the implied X-ray luminosity at the peak is in the interval $(4-20) \times 10^{36} \mathrm{erg} \mathrm{s}^{-1}$ which is effectively representative of the brightest (and rarest) outbursts typically detected from SFXTs. This is particularly true for the highest luminosity value of the order of $\sim 10^{37}$ erg $\mathrm{s}^{-1}$, which tends to favour a large distance of the order of $10 \mathrm{kpc}$. A huge number of NIR/optical sources fall 
within the $3.8^{\prime}$ error circle radius of IGR J20344+3913: 363 2MASS sources and 130 USNO-B1.0 stars. Among them, several could be the donor star in a HMXB. For instance, 2MASS $20342790+3913165$ is one of the brightest NIR sources $(\mathrm{J}=10.583 \mathrm{mag}, \mathrm{H}=9.595, \mathrm{~K}=9.262 \mathrm{mag})$ and it is located at $0.3^{\prime}$ from the IGR J20344+3913 best position. Its properties would be consistent with a B-type supergiant star located at a distance of $7.6 \mathrm{kpc}$ suffering an extinction of $\mathrm{Av}=8.6 \mathrm{mag}$. Conversely, if we assume that it is a main sequence B star, then it should be located at about $1.9 \mathrm{kpc}$ (with $\mathrm{Av}=6.5 \mathrm{mag}$ ). In conclusion, a SFXT is a viable scenario but unfortunately the large ISGRI error circle prevents us to pinpoint the correct NIR/optical counterpart and so we are unable to constrain the source nature. To this aim it is mandatory to reduce the value of the error circle radius to arcsecond size. Additionally, we note the intriguing presence of a strong radio source (listed in both NVSS and Green Bank catalogs) inside the IBIS/ISGRI error circle of this candidate SFXT. However, the possibility that such association could be simply spurious must be taken into account, and to this aim we calculated the probability of finding a NVSS radio source inside the IBIS/ISGRI error circle by chance. Given the spatial density of NVSS radio sources (as taken from Condon et al. 1998), we estimated a probability of $\sim 0.5$ chance coincidences. Such value is not low enough to strongly support a real physical association, conversely it is likely that the radio source is unrelated to IGR J20344+3913.

As extensively discussed in the previous case of IGR J03346+4414, the interesting scenario of a nearby flare star is viable for IGR J20344+3913 as well. Its spectral characteristics (soft power law with $\Gamma \sim 2.9$ and Bremsstrahlung with temperature $\mathrm{kT} \sim 28 \mathrm{keV})$, plasma temperature $(\mathrm{T} \sim$ $3 \times 10^{8} \mathrm{~K}$ ), flux/luminosity value, duration of the flaring activity, are all compatible with expectations from this interpretation.

An alternative Galactic scenario is that involving Symbiotic X-ray binaries (SyXBs). They are a subclass of low mass X-ray binaries in which material is accreted onto a neutron star compact object from the wind of a late type $\mathrm{M}$ giant companion. Only a few SyXBs are known to date (see list in Masetti et al. 2007, Kuranov \& Postnov 2015), they are mainly characterized by relatively low X-ray luminosity both at soft and hard X-rays (typically $\mathrm{L}_{x} \sim 10^{32}-10^{35}$ erg $\mathrm{s}^{-1}$ ) and show long as well as short term X-ray variability. Occasionally, they can exhibit flaring activity typical of neutron stars accreting matter from a stellar wind. The majority of known SyXBs have been detected by INTEGRAL as persistent hard X-ray sources, as such they are listed in several IBIS catalogs published to date (e.g. Bird et al. 2007, 2010, 2016) with typical fluxes of the order of $\sim 10^{-10}-10^{-11} \mathrm{erg} \mathrm{cm}^{-2} \mathrm{~s}^{-1}(20-40 \mathrm{keV})$. This is at odds with the INTEGRAL/IBIS non detection of persistent emission from IGR J20344+3913 (20-40 keV upper limit of $\left.1.1 \times 10^{-12} \mathrm{erg} \mathrm{cm}^{-2} \mathrm{~s}^{-1}\right)$ despite its sky region has been extensively exposed (i.e. the source is not listed in the latest published INTEGRAL/IBIS catalog Bird et al. 2016). In addition, the extremely short and energetic flare observed from IGR J20344+3913 is not consistent with expectations for typical duration observed for SyXBs in the hard X-ray band (i.e. from several days on).

Alternatively IGR J20344+3913 could be a blazar be- hind the Galactic plane, hypothesis supported by the presence of a bright radio source inside its error circle $(420 \mathrm{mJy}$ at $6 \mathrm{~cm}$ ) as listed in the Green Bank radio catalog. The Swift/XRT flux upper limit $\left(2.2 \times 10^{-12} \mathrm{erg} \mathrm{cm}^{-2} \mathrm{~s}^{-1}\right)$ is compatible with this blazar interpretation since it is notably in the range of soft X-ray flux values $(0.6-8) \times 10^{-12}$ erg $\mathrm{cm}^{-2} \mathrm{~s}^{-1}$ typically measured from blazars detected by INTEGRAL (Malizia et al. 2016), and therefore it is likely that even small decrements of the X-ray flux (which is likely to happen given the variable nature of blazar) could have been sufficient to hamper the detection by Swift/XRT in a very short exposure. However there are several shortcomings for such blazar scenario: i) as reported above, it is likely that the association between IGR J20344+3913 and the radio source is spurious; ii) the rather short duration of the IBIS/ISGRI flare $(\sim 30$ minutes $)$ would be among the shortest flaring episodes ever detected at hard X-rays from a blazar. In the literature, only one similar case is reported, i.e. $\mathrm{a} \sim 30$ minutes flare from a firm blazar likely detected by INTEGRAL (Foschini et al 2006); iii) our reported dynamic range of IGR J20344+3913 at hard X-rays (about three orders of magnitude) is extreme if compared to blazars, in fact as from observations of blazar X-ray variability their typical dynamic range is fairly small (from a few to a very few tens in the X-ray band). Higher blazar dynamic range values (up to about two orders of magnitude) have been achieved at gamma-ray energies only in very exceptional cases, like the remarkable blazar 3C 454.3 which is to date the most variable and bright gamma-ray blazar detected by both Fermi and AGILE (Vercellone 2012).

\section{CONCLUSIONS}

We reported on the discovery of two new Fast X-ray Transients (FXTs) from analysis of archival INTEGRAL data. The main spectral and temporal characteristics of both IGR J03346+4414 and IGR J20344+3913 are best compatible with a Galactic origin such as a SFXT or a nearby flare star. Conversely, the extragalactic blazar scenario presents some shortcomings. Regardless of their nature, their peculiar characteristics (e.g. high plasma temperature for IGR J03346+4414) as well as their unusually short and bright outbursts make them particularly interesting. This kind of source is very difficult to discover and characterize due to the very transitory nature and especially the very low duty cycle. The instrumental characteristics of IBIS/ISGRI onboard INTEGRAL are particularly suited in serendipitously detecting and discovering such short duration random events. It seems plausible that other such sources wait to be discovered, further exploitations of the entire INTEGRAL data archive may yield additional discoveries of this kind of interesting X-ray transients.

\section{ACKNOWLEDGMENTS}

We thank the anonymous referee for useful comments which helped us to improve the quality of this paper. We thank the Swift team, the PI, the duty scientists and science planners for making the two ToO observations reported here 
possible. We acknowledge financial support from the Italian Space Agency via INTEGRAL ASI/INAF agreement n. 2013-025.R.0, and the grant from PRIN-INAF 2014, "Towards a unified picture of accretion in High Mass X-Ray Binaries". This work has made use of the INTEGRAL archive developed at INAF-IASF Milano (http://www.iasfmilano.inaf.it/ ada/GOLIA.html). This research has made use of data and/or software provided by the High Energy Astrophysics Science Archive Research Center (HEASARC), which is a service of the Astrophysics Science Division at NASA/GSFC and the High Energy Astrophysics Division of the Smithsonian Astrophysical Observatory.

\section{REFERENCES}

Bird, A. J., Malizia, A., Bazzano, A., et al. 2007, ApJS, 170, 175 Bird, A. J., Bazzano, A., Bassani, L., et al. 2010, ApJS, 186, 1

Bird, A. J., Bazzano, A., Malizia, A., et al. 2016, ApJS, 223, 15

Condon J.J., Cotton W. D., Greisen E.W., et al. 1998, AJ, 115, 1693

Copete, A. Grindlay J., Barthelmy, S., 2008, GCN circular 8375

Cutri R. M., Skrutskie M. F., van Diyk S., et al. 2003, VizieR Online data Catalog, 2246

Dickey J. M., Lockman F. J., 1990, ARA\&A, 28, 215

Foschini, L., Pian, E., Maraschi, L., et al. 2006, A\&A, 450, 77

Getman, K. V., Feigelson, E. D., Broos, P. S., et al. 2008, ApJ, 688,418

Gregory P.C., Scott W. K., Douglas K., et al. 1996, ApJS, 103, 427

Hachisuka, K., Brunthaler, A., Menten, K. M., et al. 2009, ApJ, 696,1981

Hudson, H. S., Nitta, N., 1996, AIPC, 374, 285

Kothes, R., Sun, X. H., Reich, W., et al. 2014, ApJ, 784L, 26

Krivonos, R., Revnivtsev, M., Tsygankov, S., et al., 2010, A\&A, 519,107

Kuranov, A. G., Postnov, K. A., 2015, AstL, 41, 114

Lasker B. M., Lattanzi M. G., McLean B. J., et al. 2008, AJ, 136, 735

Lebrun F., et al., A\&A 2003, 411, 141

Maccacaro T., Gioia, I. M., Wolter A., et al., 1988, ApJ, 326, 680

Maggio, A., 2008, MmSAI, 79, 186

Malizia, A., Landi, R., Molina, M., et al. 2016, in press, astroph 1604.06303

Masetti, N., Landi, R., Pretorius, M. L., et al. 2007, A\&A, 470, 331

Monet D. G., Levine S. E., Canzian B., et al. 2003, AJ, 125, 984

Negueruela I., Smith D., Reig P., et al. 2006, ESA SP-604: X-ray Universe 2005, Wilson A. editor, 2006, 165

Osten, A. R., et al. 2007, ApJ, 654, 1052

Osten, A. R., et al. 2010, ApJ, 721, 785

Osten, A. R., et al. 2016, AAS Meeting \#227, id.145.17

Paizis, A., et al. 2013, Astronomy \& Computing, 1, 33

Paizis, A. \& Sidoli L., 2014, MNRAS, 439, 3439

Pallavicini, R., Tagliaferri, G., Maggio, A., 2000, AdSpR, 25, 517

Sguera, V., Barlow, E. J., Bird, A. J., et al. 2005, A\&A, 444, 221

Sguera, V., Bazzano, A., Bird, A. J., et al. 2006, ApJ, 646, 452

Sguera, V., Bassani, L., Landi, R., et al. 2008, A\&A, 487, 619

Sguera, V., Drave, S. P., Sidoli, L., et al. 2013, A\&A, 556, 27

Schmitt J. H. M. M. \& Favata F., 1999, Nature 401, 44

Ubertini P., et al., 2003, A\&A, 411, L131

Vercellone, A., 2012 Fermi \& Jansky Proceedings, arXiv 1205.5510

Winkler C., et al., 2003, A\&A, 411, L1 